\title{
Diálisis peritoneal automática adaptada: un método de prescripción eficaz, eficiente y seguro
}

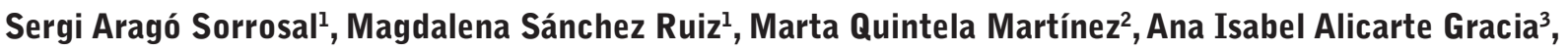 \\ Manel Vera Rivera ${ }^{4}$
}

${ }^{1}$ Diplomado en enfermería, ${ }^{2}$ Cordinadora enfermería de diálisis, ${ }^{3}$ Directora de Enfermería, ${ }^{4}$ Nefrólogo, Unidad de Diálisis Peritoneal. Servicio de Nefrología y Trasplante Renal. Instituto Clínico de Nefrología y Urología. Hospital Clínic de Barcelona

\section{Resumen}

El objetivo del trabajo es analizar los resultados obtenidos tras la introducción de la modalidad de diálisis peritoneal automática adaptada, usando bicarbonato puro como agente tampón, en un grupo de pacientes en programa de diálisis peritoneal automática convencional.

Estudio de diseño cuasi experimental, que se realizó entre los meses de febrero y diciembre de 2013, en la unidad de diálisis peritoneal del Hospital Clínic de Barcelona.

Los sujetos de estudio fueron 12 pacientes, 7 mujeres y 5 hombres, de dicha unidad en modalidad de diálisis peritoneal automática convencional. Edad media $58 \pm 12$ años (rango: 34-71).

El estudio consistió en comparar dos modalidades de diálisis peritoneal automática. Se inició el estudio con prescripción de diálisis convencional, durante tres meses, para cambiar a modalidad de diálisis adaptada durante el mismo intervalo de tiempo. Y finalizarlo, con tres meses de pauta de diálisis convencional. Se prescribió el mismo volumen total de líquido de diálisis y tiempo de sesión, para cada paciente en ambas modalidades, variando los volúmenes y tiempos de permanencia en función de la modalidad. Se utilizó solución de diálisis con bicarbonato puro y glucosa $1,5 \%$. Todos los pacientes, con día seco.

Se monitorizaron las variables al inicio del estudio, y en cada cambio de prescripción.

Los resultados obtenidos en diálisis convencional: $\mathrm{Kt} /$ $v=2,3 \pm 0,2$, aclaramiento de creatinina $=63 \pm 8$ litros $/ \mathrm{se}$ -

Correspondencia:

Sergi Aragó Sorrosal.

Servicio de Nefrología y Trasplante Renal

Hospital Clínico de Barcelona

$\mathrm{C} /$ Villarroel $\mathrm{n}^{\circ} 170$

08036. Barcelona

E-mail:sarago@clinic.ub.es mana, y ultrafiltración $=842 \pm 110 \mathrm{ml}$. En diálisis adaptada, $\mathrm{Kt} / \mathrm{v}=2,8 \pm 0,2$, aclaramiento de creatinina $=74 \pm 9$ litros/ semana, y ultrafiltración $=982 \pm 123 \mathrm{ml}$.

La diálisis adaptada permite obtener una adecuada eficacia de tratamiento, mejora los parámetros de adecuación de diálisis y ultrafiltración; siendo segura y cómoda para el paciente.

\author{
PALABRAS CLAVE \\ - PRESCRIPCIÓN DIÁLISIS \\ - EFICACIA DIÁLISIS PERITONEAL \\ - ADAPTADA \\ - VOLÚMENES \\ - PERMANENCIA
}

Adapted automated peritoneal dialysis: an effective, efficient and safe prescription method

\section{Abstract}

The aim of this study is to analyze results obtained following the introduction of the adapted automated peritoneal dialysis, in a group of patients in conventional automated peritoneal dialysis program using bicarbonate-buffered dialysate.

Design trial in experimental phase, which was conducted in the months from February and December 2013, in the unit of peritoneal dialysis in the Hospital Clinic of Barcelona. Study subjects were 12 patients, 7 women and 5 men, in conventional automated peritoneal dialysis modality. Mean age $58 \pm 12$ years (range: $34-71$ ).

The study assessed two automated peritoneal dialysis prescriptions. This study was started with conventional 
dialysis for three months. Then, dialysis prescription was modified, and adapted was applied during same interval of time. And finally, conventional dialysis for three months. The same total volume of dialysate, the same duration, and the same $1,5 \%$ glucose bicarbonatebuffered dialysate, were prescribed in both modalities. Only dwell time and fill volume were modified, when dialysis modality was changed. All patients, on dry day. Variables at the beginning of the study, and at each change of prescription were seldom monitored.

Outcomes for conventional dialysis: Kt/ $v=2,3 \pm 0,2$, creatinine clearance $=63 \pm 81 /$ week, and ultrafiltration $=842 \pm 110 \mathrm{ml}$. For dialysis adapted, Kt/ $v=2,8 \pm 0,2$, creatinine clearance $=74 \pm 91 /$ week, and ultrafiltration $=982 \pm 123 \mathrm{ml}$.

Adapted dialysis allows appropriate treatment efficiency, improves adequacy of dialysis and ultrafiltration parameters; being safe and comfortable for the patient.

\section{KEYWORDS}

- DIALYSIS PRESCRIPTION

- PERITONEAL DIALYSIS EFFICIENCY

- ADAPTED

- VOLUMES

- DWELL

\section{Introducción}

La diálisis peritoneal (DP) es una de las terapias renales sustitutivas (TRS), junto a la hemodiálisis (HD) y el trasplante renal (TxR), y uno de los tratamientos ambulatorios por excelencia. Existen dos modalidades distintas de DP: la diálisis peritoneal continua ambulatoria (DPCA) y la diálisis peritoneal automatizada (DPA). En la DPCA, el tratamiento consiste en realizar un determinado número de intercambios diarios por el paciente, habitualmente entre 3 y 5 , con permanencias largas $^{(1)}$. Por contra, la DPA se basa en la conexión del paciente a una cicladora durante 8-9 horas, y la realización de varios intercambios de igual duración, con permanencias más cortas que en la DPCA. En ocasiones, con día seco, la cavidad peritoneal del paciente está vacía durante el día; o en tratamiento de diálisis peritoneal continua cíclica, se infunde una cierta cantidad de volumen en la cavidad peritoneal del paciente durante el día ${ }^{(2)}$. En ambas modalidades, el volumen de llenado suele ser el mismo en todas las permanencias ${ }^{(1-3)}$.

Alcanzar una correcta adecuación en DP supone ir más allá de obtener un $\mathrm{Kt} / \mathrm{v}$ semanal de urea $\geq 1,7$ o un acla- ramiento semanal de creatinina $\geq 50$ litros. Es preciso mantener una correcta ultrafiltración (UF), conseguir un estado de hidratación óptimo, un control adecuado de la presión arterial, y todo ello con el menor coste metabólico para conseguir una terapia eficaz, como demuestran algunos trabajos ${ }^{(4-5)}$.

Estos requisitos obligan a individualizar el tratamiento para cada paciente con las herramientas que se tienen a disposición, junto a la prescripción de DP. Estas herramientas son la prueba de equilibrio peritoneal (PEP), y la medición de la presión hidrostática intraperitoneal (PIP). Ambas han sido ampliamente descritas en la literatura $^{(6-9)}$.

Siguiendo las modalidades antes descritas, DPA y DPCA, este objetivo no será fácil de alcanzar; ya que la eficacia de la DP dependerá de modificar los tiempos y los volúmenes de las permanencias. Como fue descrito en 1994, por Fischbach et al, "permanencias cortas favorecen la UF, a la vez que permanencias largas mejoran las depuración de solutos" (5).

De esta forma, junto a la información que aportan la PEP y la medición de la PIP, Fischbach et al ha propuesto un nuevo método para la prescripción de la DPA. Éste se basa en una sucesión de ciclos de permanencias cortas y bajo volumen de llenado, que preceden a ciclos más largos y mayor volumen infundido; a diferencia de la forma habitual de pauta de DPA que se ha descrito previamente ${ }^{(3)}$.

¿Y qué se consigue con ello? Está demostrado que el tiempo de permanencia y el volumen de llenado afectan a la eficacia de la DP. De esta forma, mediante permanencias cortas se consigue una correcta UF, ya que el gradiente osmótico proporcionado por la glucosa desaparece a medida que transcurre el tiempo, perdiendo su capacidad de UF. Pero no son efectivas para la depuración de solutos. Hecho que se compensa con las permanencias más largas, que favorecen dicha depuración ${ }^{(3,5-6,10)}$.

Siguiendo con esta prescripción, mayores volúmenes de llenado, provocan que la superficie de la membrana peritoneal en contacto con el líquido peritoneal sea mayor, consiguiendo una mejor eliminación de toxinas urémicas. Por consiguiente, volúmenes menores logran una UF más efectiva, debido a una presión intraperitoneal menor y a una disminución de la reabsorción del líquido de diálisis $(3,6,11)$.

Esta nueva forma de pautar la DPA ha sido denominada "adapted automated peritoneal dialysis" (APD-A), diálisis peritoneal automatizada adaptada, por Fischbach et al. Consiste en la prescripción de varios ciclos con diferen- 
tes tiempos de permanencia y volúmenes infundidos. Se pautan ciclos de permanencias cortas, con volúmenes de llenado pequeños, que favorecen la UF. Seguidos de otros ciclos con permanencias más largas, y mayores volúmenes que dan lugar a una mejor depuración de solutos (figura 1). Los tiempos se prescriben a partir del tiempo APEX (accelerated peritoneal equilibration point: el punto en que la curva de saturación de urea se cruza con la de disminución de la concentración de glucosa) y el tiempo óptimo de depuración de fosfatos (TDF), determinado por el punto en que el D/P de fosfato es igual a 0,6 y que equivale a 3-4 veces el valor del tiempo APEX; ambos obtenidos en la PEP (figura 2). Mientras que los volúmenes son prescritos en función de la superfície corporal del paciente ${ }^{(3,5)}$.
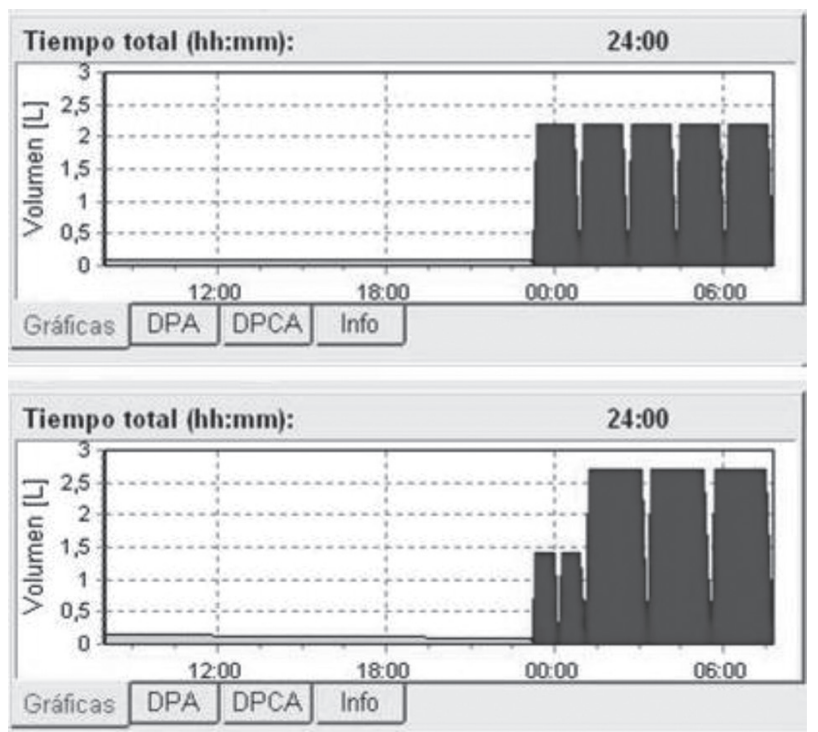

Figura 1. Prescripciones para diálisis peritoneal automatizada.

En la imagen superior, diálisis peritoneal automatizada convencional; 5 permanencias, del mismo volumen cada una. Debajo, pauta de diálisis peritoneal automatizada adaptada; 2 ciclos cortos con poco volumen de infundido, seguidos de tres ciclos de mayor volumen.

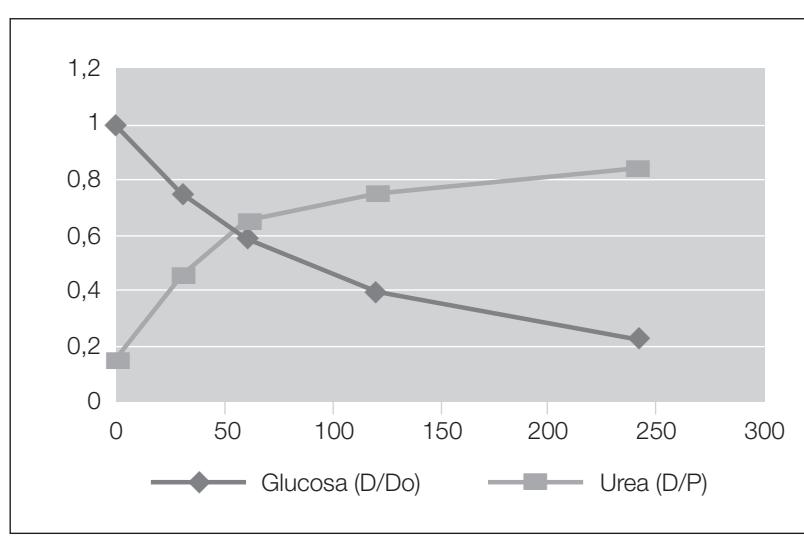

Figura 2. Tiempo APEX (accelerated peritoneal equilibration point: el punto en que la curva de saturación de urea se cruza con la de disminución de la concentración de glucosa).
La realización de esta modalidad de DPA requiere de una cicladora que permita individualizar las características de volumen y permanencia de cada ciclo.

En los trabajos realizados hasta ahora sobre la APD-A, la técnica ha sido descrita en pacientes adultos y pediátricos, sólo con líquidos de diálisis con tampón lactato y glucosa baja; aportando buenos resultados, en términos de UF y depuración de solutos ${ }^{(3,10)}$. Otras experiencias en el tratamiento personalizado de la DPA en adultos han sido descritas ${ }^{(12)}$.

En este artículo, reproduciendo la experiencia de Fischbach et al, se comparan ambas técnicas, DPA-adaptada (DPAa) y DPA-convencional (DPAc), en un grupo de pacientes de la unidad de diálisis peritoneal del Hospital Clínic, mediante el uso de líquidos de tampón bicarbonato y glucosa baja.

\section{Objetivos}

Objetivo general:

- Analizar los resultados obtenidos tras la introducción de la modalidad de diálisis peritoneal automática adaptada, usando bicarbonato puro como agente tampón, en un grupo de pacientes en programa de diálisis peritoneal automática convencional.

\section{Como objetivos específicos:}

- Identificar posibles complicaciones en los pacientes de DP, usando una nueva forma de prescripción.

- Identificar la carga de trabajo que supone para Enfermería la implementación de un nuevo método de prescripción en DPA.

\section{Material y métodos}

Estudio de diseño cuasi experimental, que se llevó a cabo entre los meses de febrero a diciembre de 2013. El ámbito en el que se realizó fue la unidad de DP del Hospital Clínic de Barcelona.

La población de estudio fueron los pacientes de dicha unidad. Se usó una técnica de muestreo no probabilística: consecutiva; y no hubo randomización. Los sujetos de estudio fueron su propio control. Los criterios de selección fueron los siguientes:

- Criterios de inclusión: modalidad de DPA para el estudio durante un tiempo mínimo de 3 meses, no presentar episodios de peritonitis previo a la inclu- 
sión en el ensayo, realización de PEP para determinar transporte peritoneal (medio-alto).

- Criterios de exclusión: rechazo a participar en el estudio, presencia de patología abdominal o respiratoria que pudiera ser agravada por volúmenes de infusión altos, disfunción del catéter peritoneal.

La muestra estaba formada por 7 mujeres (58\%), y 5 hombres (42\%). Edad media 58 \pm 12 años (rango: 3471). Las causas de la insuficiencia renal fueron: nefropatía diabética (3), nefroangioesclerosis (5), nefropatía intersticial (1), nefropatía glomerular (1), y etiología desconocida (2). Destacar que tres pacientes presentaban diabetes mellitus tipo II, y 9 pacientes requerían medicación hipotensora. Todos ellos conservaban función renal residual. Previo al inicio del estudio, se realizó una PEP a cada uno de los sujetos, con determinación del tipo de transporte peritoneal y el tiempo APEX.

Una vez incluidos en el ensayo, se enrolaba a los pacientes durante nueve meses, en los que se iba a aplicar las dos pautas de DPA a intervalos de 3 meses. Empezaron con una prescripción de DPAcl durante el período de tiempo planificado. Pasado este tiempo, realizaron el tratamiento de DPAa2 durante el mismo intervalo. Para finalizar, realizando los últimos tres meses con pauta de DPAc3. En ambas modalidades, se prescribió el mismo volumen total de líquido de diálisis, tiempo de sesión, y día seco.

Para la prescripción del tiempo total de cada una de las pautas, se utilizó el tiempo APEX obtenido en la PEP de cada uno de los pacientes.

En la modalidad de DPAa, fueron prescritos 2 ciclos iniciales de corta duración; y tres ciclos largos.

Para la determinación del volumen de solución de diálisis de las permanencias, se calculó mediante el índice de superficie corporal (BSA) de cada paciente; en función de la fórmula: $1500 \mathrm{ml} / \mathrm{m} 2$ multiplicado por cuatro. En la DPAc, se infundió el mismo volumen en todos los ciclos. Mientras que en la prescripción de DPAa, en los ciclos cortos, se infundían volumen menores que en los ciclos largos.

La duración media de las sesiones de diálisis fue de $522 \pm 76$ minutos y la media del volumen total de solución utilizado fue de 10,3 $\pm 2,3$ litros.

Se utilizó sólo solución de diálisis peritoneal de tampón bicarbonato y glucosa al 1,5\%.

Todos los pacientes finalizaron el estudio.
Las variables monitorizadas fueron:

- Peso.

- Tensión arterial.

- Estado de hidratación mediante Bioimpedancia multifrecuencia (BIA).

- Parámetros analíticos (bioquímica y hemograma).

- Parámetros de adecuación (aclaramiento de creatinina $\mathrm{y} \mathrm{Kt} / \mathrm{v}$ ).

Se realizó monitorización de las variables previo al inicio del estudio. Y tras cada cambio de prescripción; a los tres meses, a los seis meses y a los nueve meses.

Se realizó un seguimiento de los datos registrados en la tarjeta de la cicladora. Tiempos, permanencias y alarmas, en ambas prescripciones. Y seguimiento telefónico de los pacientes, para descartar posibles complicaciones.

\section{Resultados}

El programa utilizado para el análisis de los resultados ha sido IBM SPSS vr 21 (Windows).

Para el análisis descriptivo, los resultados están expresados en media y desviación estándar.

Después de la $\mathrm{DPAa}_{2^{\prime}}$ el resultado para la media del

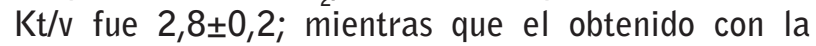
$\mathrm{DPAc}_{3}$ fue de $22,3 \pm 0,2$. La UF media conseguida en la DPAa2 fue de $982 \pm 123 \mathrm{ml}$; en la DPAc3, $842 \pm 110 \mathrm{ml}$. El aclaramiento semanal de creatinina para la $\mathrm{DPAa}_{2}$ fue de $74 \pm 9$ itros/semana; en el caso de la DPAc ${ }_{3}$ al-

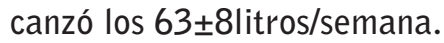

Se describieron las medias de los valores previos al inicio del estudio, y después de la prescripción de $\mathrm{DPAa}_{2}$ y $\mathrm{DPAc}_{3}$ para la tensión arterial, el peso y la bioimpedancia (tabla 1).

Tabla 1.

\begin{tabular}{|l|r|r|r|}
\hline Variables $(\mathbf{n = 1 2})$ & \multicolumn{1}{|c|}{ Inicio } & \multicolumn{1}{c|}{ DPAa $_{2}$} & \multicolumn{1}{c|}{ DPAC $_{3}$} \\
\hline Peso & $66,5 \pm 11,6$ & $67,02 \pm 11,7$ & $67,64 \pm 12$ \\
& $84-41,7$ & $86-41,9$ & $88-42,8$ \\
\hline \multirow{2}{*}{ Tensión arterial sistólica } & $130,25 \pm 26,2$ & $139,58 \pm 25,5$ & $134,83 \pm 19,1$ \\
& $173-86$ & $177-90$ & $163-101$ \\
\hline \multirow{2}{*}{ Tensión arterial diastólica } & $76,33 \pm 8,5$ & $82,16 \pm 12,4$ & $80,75 \pm 11,4$ \\
& $93-60$ & $102-66$ & $101-66$ \\
\hline Bioimpedancia & $1,11 \pm 1.2$ & $1,11 \pm 0,9$ & $1,1 \pm 1.1$ \\
& $3,31-0,9$ & $2,4 /-0,4$ & $2,8 /-0,8$ \\
\hline
\end{tabular}

Valores expresados en medias, desviación estándar y rangos de los valores previos al inicio del estudio, y después de la prescripción de DPAa y DPAc. 
Se comprobaron las alarmas durante las tres fases de la DPA (tabla 2).

Tabla 2.

\begin{tabular}{|c|c|c|c|}
\hline & DPAC $_{1}$ & DPAa $_{2}$ & DPAC $_{3}$ \\
\hline & $n=12$ & $n=12$ & $n=12$ \\
\hline Media & $61,58 \pm 6,9$ & $63,16 \pm 9,3$ & $65,5 \pm 8.4$ \\
\hline Rango & $70-51$ & $75-47$ & $89-54$ \\
\hline
\end{tabular}

Valores expresados en medias y rangos de las alarmas al inicio del estudio, y después de la prescripción de DPAa y DPAc.

La media de la hemoglobina glicada $(\mathrm{HbAc})$ para la DPAa2 fue $5,24 \pm 0,52$; y para la DPAc3, 5,48 $\pm 0,56$. Ver resultados en tabla 3 y figuras 3-4.

Tabla 3.

\begin{tabular}{|c|c|c|c|c|}
\hline Paciente & HbAlc $_{\mathbf{0}}$ & HbAlc $_{1}$ & HbAlc $_{2}$ & HbAlc $_{3}$ \\
\hline $\mathbf{1}$ & 5,4 & 5,3 & 5,1 & 5,3 \\
\hline $\mathbf{2}$ & 4,9 & 4,9 & 4,7 & 4,8 \\
\hline $\mathbf{3}$ & 4,8 & 5 & 4,6 & 4,9 \\
\hline $\mathbf{4}$ & 5,3 & 5,1 & 5 & 5,2 \\
\hline $\mathbf{5}$ & 5,3 & 5,3 & 5,1 & 5,4 \\
\hline $\mathbf{6}$ & 5,6 & 5,5 & 5,2 & 5,5 \\
\hline $\mathbf{7}$ & 5,1 & 5 & 4,8 & 5 \\
\hline $\mathbf{8}$ & 5,3 & 5,3 & 5,1 & 5,3 \\
\hline $\mathbf{9}$ & 5,5 & 5,6 & 5,2 & 5,4 \\
\hline $\mathbf{1 0}$ & 6,3 & 6,2 & 5,8 & 6,1 \\
\hline $\mathbf{1 1}$ & 6,7 & 6,6 & 6,2 & 6,5 \\
\hline $\mathbf{1 2}$ & 6,6 & 6,5 & 6,1 & 6,4 \\
\hline
\end{tabular}

Valores Hemoglobina glicada durante el estudio.

$\mathrm{HbAlC}_{0}$, resultado previo a la inclusión.

$\mathrm{HbAlc}_{1}$, resultado después de la pauta DPAc . $_{1}$. $\mathrm{HbAlc}_{2^{\prime}}$ resultado después de la pauta DPAa ${ }_{2}$. $\mathrm{HbAlC}_{3}$, resultado después de la pauta DPAc ${ }_{3}$.

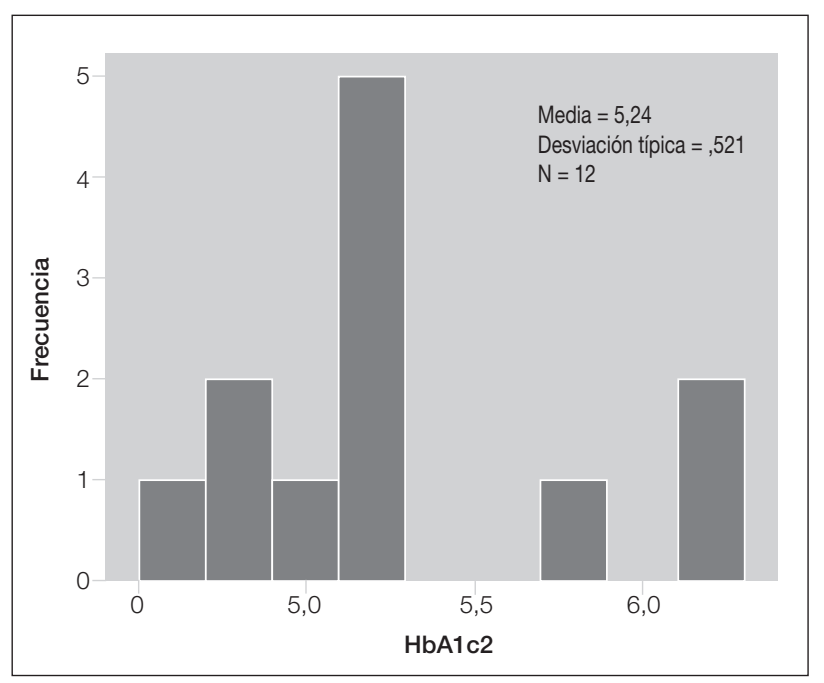

Figura 3. Resultado HbAlc en $\mathrm{DPAa}_{2}$.

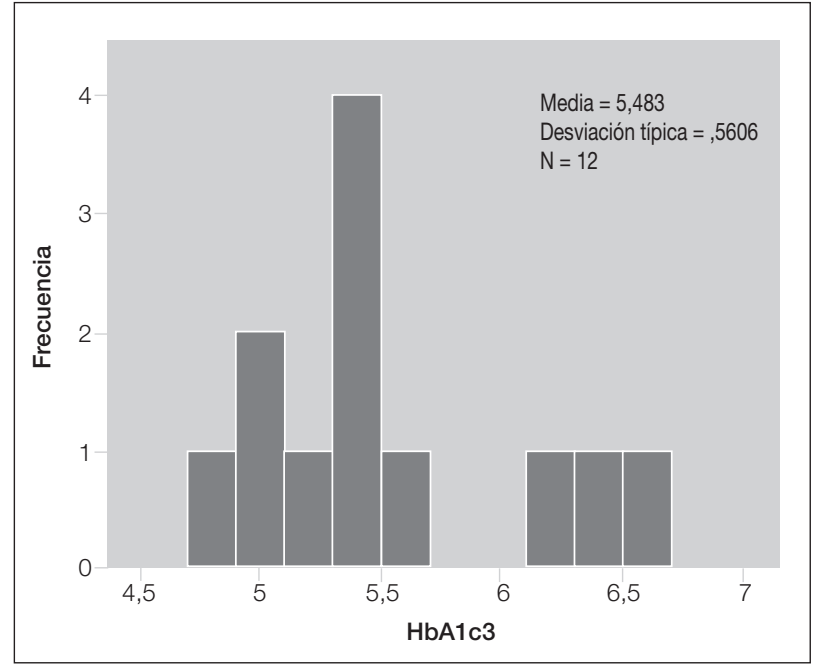

Figura 4. Resultado $\mathrm{HbAlc}$ en $\mathrm{DPAc}_{3}$.

\section{Discusión}

Se ha estudiado que los pacientes en tratamiento de DPCA presentan mayor depuración de toxinas urémicas y eliminación de sodio, que aquéllos que realizan su tratamiento en DPA ${ }^{(3,13-14)}$.

Entonces, ¿qué ocurre cuando un paciente elige la modalidad de DPA?

Del trabajo de enfermería nefrológica y el equipo médico de nefrología, depende que los pacientes en DP puedan conciliar las actividades de su vida diaria con su tratamiento; sin que dicho tratamiento pierda eficacia.

Y la prescripción de DP no sólo ha de proporcionar unos parámetros óptimos de adecuación, también tiene que asegurar una correcta UF; y debe ser personalizada al paciente, en función de los hábitos de vida, y la evolución clínica del mismo ${ }^{(3-5,12)}$.

En esta línea, Fischbach et al describió una nueva manera de prescribir la DPA, la DPA adaptada; variando tiempos y volúmenes de permanencia para optimizar el tratamiento. Con ello, se consiguió mejorar parámetros como la depuración de toxinas urémicas, y la eliminación de sodio; utilizando permanencias largas con volúmenes altos, para promover el aclaramiento de solutos; y permanencias cortas junto a volúmenes de infusión pequeños, que mejoraron la UF. También describió un descenso en las cifras de la tensión arterial de los pacientes ${ }^{(3,10)}$.

En este estudio, se reprodujo la experiencia de Fischbach et al, usando líquido con tampón bicarbonato. Comparando la DPAc con la DPAa, se obtuvieron mejores resultados 
en parámetros como Kt/v, UF, y aclaramiento semanal de creatinina en la prescripción de DPAa. Se observó una disminución de los valores de la hemoglobina glicada $(\mathrm{HbAlc})$; dando lugar a la hipótesis, que la DPAa resulta una buena opción para lograr mayor UF, con menor absorción de glucosa. No se detectaron diferencias en el control de la tensión arterial ni en la composición corporal de los pacientes, mediante BIA.

El uso de volúmenes diferentes de infusión y las consecuencias que pueden derivarse de ello han sido descritas; pero aún son necesarios más estudios ${ }^{(15)}$. En el grupo de Fischbach, no se detectaron complicaciones a consecuencia de los altos volúmenes usados en las permanencias largas de difusión; en este estudio, los hallazgos, tampoco fueron significativos. Tan sólo en uno de los casos, hubo que modificar el volumen de las permanencias largas para difusión por referir molestias, y sensación de plenitud, durante la sesión.

Se utilizó bicarbonato como agente tampón, para comprobar la UF conseguida, debido a la hipótesis que el uso de estos líquidos puede dar lugar a una disminución de la UF, compensada por un aumento del volumen residual de diuresis (16). Hipótesis que ha sido discutida recientemente en la revisión de Cho et al ${ }^{(17)}$.

Cabe destacar varias limitaciones en el estudio. Entre ellas, el tamaño de la muestra, hecho que provoca que el estudio no pueda poseer validez externa; no se ha realizado randomización para el estudio, ni análisis inferencial debido al número de sujetos; se ha estudiado sólo en pacientes con transporte peritoneal medio-alto; y no se midió la presión intraperitoneal en ningún sujeto.

\section{Conclusiones}

El estudio permite observar, que para la muestra estudiada, la DPAa obtiene una adecuada eficacia de tratamiento, mejora los parámetros de adecuación de diálisis y ultrafiltración; sin poder demostrar cambios en el estado de hidratación, ni del control de las cifras de tensión arterial. No hay aumento significativo de las alarmas y tiempo. Por tanto, la DPAa es una modalidad segura y eficiente para el paciente; y puede sugerirse que podría mejorar el control glicémico, al precisar menor absorción de glucosa para mantener la UF.

Pero estos resultados no son concluyentes; y se necesitan nuevos estudios, multicéntricos y más extensos para conocer los efectos a largo plazo de la aplicación de esta nueva prescripción; por ejemplo, complicaciones en la pared abdominal, o sesiones demasiado extensas por tiempos de drenaje aumentados derivadas del uso de volúmenes de llenado altos.

Este trabajo fue aprobado por el Comité de Ética e Investigación del Hospital Clínic. Todos los participantes fueron informados del estudio; participaron por voluntad propia, y firmaron un consentimiento informado.

Los autores del presente trabajo declaran que no existe ningún conflicto de intereses.

\section{Agradecimientos}

A todos los pacientes de nuestra unidad, en especial, a los que tomaron parte en el estudio. Al equipo de $\mathrm{Ne}-$ frología y a las personas que nos han apoyado. Sin todos ellos no hubiera sido posible este trabajo.
Recibido: 15 Agosto 2014
Revisado: 18 Agosto 2014
Modificado: 18 Agosto 2014
Aceptado: 18 Agosto 2014

\section{Bibliografía}

1. López JM. Diálisis peritoneal continua ambulatoria. En: Montenegro J, Correa-Rotter R, Riella MC, Eds. Tratado de diálisis peritoneal. Elsevier. España. Barcelona, $3^{\circ}$ Ed, 2009: 165-185.

2. Caravaca, F. Diálisis peritoneal automatizada. En: Montenegro J, Correa-Rotter R, Riella MC, Eds. Tratado de diálisis peritoneal. Elsevier. España. Barcelona, $3^{\circ} \mathrm{Ed}, 2009 ; 187-205$.

3. Fischbach $M$, Issad $B$, Dubois $V$, Taamma R. The beneficial influence on the effectiveness of automated peritoneal dialysis of varying the dwell time (short/long) and fill volume (small/large): a randomized controlled trial. Perit Dial Int. 2011; 31(4):450-458.

4. Lo WK, Ho YW, Li CS, Wong KS, Chan TM, YU AW, et al. Effect of Kt/v on survival and clinical outcome in CAPD patients in a randomized prospective study. Kidney Int. 2003; 64:649-656.

5. Fischbach M, Lahlou A, Eyer D, Desprez P, Geisert J. Determination of individual ultrafiltration 
time (APEX) and purification phosphate time by peritoneal equilibration test: application to individual peritoneal dialysis modality prescription in children. Perit Dial Int. 1996; 16 (suppl1):557-560.

6. Fischbach M, Zaloszyc A, Schaefer B, Schmitt CP. Optimizing peritoneal dialysis prescription for volume control: the importance of varying dwell time and dwell volume. Pediatr Nephrol. 2014; 29(8):1321-1327.

7. Fischbach $M$, Terzic J, Laugel V, Escande B, Dangelser CL, Helmstetter A. Measurement of hydrostatic intraperitoneal pressure: a useful tool for the improvement of dialysis dose prescription. Pediatr Nephrol. 2003; 18(10):976-980.

8. Rodríguez $A$, Pérez $M$. ¿Es útil la cinética peritoneal en la práctica clínica? Rev Nefrol.2013; 33(3):410-415.

9. Blasco C, Ponz E, Betancourt L, Otero S, Marquina D, Grau C, et al. Relación entre la presión intrabdominal en diálisis peritoneal con las hernias y fugas. Rev Soc Esp Enferm Nefrol. 2012; 15(2):94100.

10. Fischbach M, Desprez P, Donnars F, Hamel G, Geisert J. Optimization of CCPD Prescription in children using peritoneal equilibration test. Adv Perit Dial. 1994; 10:307-309.

11. Chagnac A, Herskovitz P, Ori Y, Weinstein T, Hirsh J, Katz $M$, et al. Effect of increased dialysate volume on peritoneal surface area among peritoneal dialysis patients. J Am Soc Nephrol. 2002; 13:2554-2559.
12. Galli E, Taietti C, Borghi M. Personalization of automated peritoneal dialysis treatment using a computer modeling system. Adv Perit Dial. 2011; 27:90-96.

13. Ortega 0 , Gallar $P$, Carreno $A$, Gutiérrez $M$, Rodríguez I, Oliet $A$, et al. Peritoneal sodium mass removal in continuous ambulatory peritoneal dialysis and automated peritoneal dialysis: influence on blood pressure control. J Am Soc Nephrol. 2011; 21:189-193.

14. Domenici A, Scabbia L, Sivo F, Falcone C, Punzo $G$, Menè $P$. Determinants of sodium removal with tidal automated peritoneal dialysis. Adv Perit Dial. 2012; 28:16-20.

15. Harris KP, Keogh AM, Alderson L. Peritoneal dialysis fill volume: can the patient tell the difference? Perit Dial Int. 2001;21(3):144-147.

16. Montenegro J, Saracho RM, Martínez IM, Muñoz RI, Ocharan JJ, Valladares E. Long-term clinical experience with pure bicarbonate peritoneal dialysis solutions. Perit Dial Int. 2006; 26:89-94.

17. Cho Y, Johnson DW, Badve SV, Craig JC, Strippoli GF, Wiggins KJ. The impact of neutral-ph peritoneal dialysates with reduced glucose degradation products on clinical outcomes in peritoneal dialysis patients. Kidney Int. 2013; 84:969-979. 